\title{
ReMed: rapport annuel 2019
}

ReMed, comité de direction

\begin{abstract}
ReMed est le réseau chargé de soutenir les médecins en cas de crise et les sensibiliser à la nécessité de préserver leur propre santé. Il est à vos côtés pour vous soutenir dans les 72 heures. Le contact peut également être établi par l'intermédiaire d'une personne de l'entourage du médecin concerné. Le principal objectif de ReMed est de maintenir le bon état de santé et la capacité fonctionnelle des médecins, afin de garantir la sécurité des patients et une qualité élevée des soins.
\end{abstract}

\section{Adapté aux spécificités des médecins - par des médecins pour les médecins}

ReMed est une organisation du corps médical financée par la Fédération des médecins suisses (FMH). La mise en œuvre opérationnelle du réseau de soutien a lieu en dehors des structures de la FMH. Le Réseau ReMed base ses procédures sur les prescriptions légales en vigueur et sur le Code de déontologie de la FMH.

L'offre ReMed - Réseau de soutien pour médecins - est fondée sur la confidentialité et la responsabilité individuelle de chaque médecin. ReMed n'intervient que si le médecin concerné y consent explicitement. Toutes les démarches sont entreprises en accord avec lui, et il bénéficie des mêmes droits que tout autre patient: secret médical et respect des dispositions sur la protection des données. ReMed n'est pas un organe de surveillance et n'a pas de compétence d'investigation ni de sanction.

Alors même que la FMH finance ReMed, elle n'a - à aucun moment - accès aux dossiers personnels des médecins qui font appel au réseau de soutien. ReMed est lié au secret médical, et les tâches médicales et administratives sont strictement séparées.

\section{Confidentialité et responsabilité}

Lorsque vous vous adressez à ReMed, vous ne prenez aucun engagement - rien ne sera entrepris sans votre consentement. Et tout ce que vous confierez à ReMed restera confidentiel: le réseau de soutien est lié au secret professionnel.

Les médecins en crise qui se tournent vers ReMed bénéficient des mêmes droits que n'importe quel autre patient: secret médical et respect des dispositions sur la protection des données. Par ailleurs, les mêmes ex- ceptions légales s'appliquent au secret médical. Seuls les collaborateurs de ReMed en charge du cas ont accès aux données personnelles du patient. Conformément à la législation sur la protection des données, les médecins qui recourent au service ont un droit de regard sur les données collectées à leur sujet.

\section{ReMed se fait connaître}

Par le biais d'articles dans la presse générale et la presse spécialisée, d'émissions radio et de témoignages publiés dans le BMS où des médecins relatent la crise qu'ils ont traversée et leur expérience avec ReMed, mais aussi de présentations faites dans les hôpitaux, les cliniques, les instituts, les sociétés de médecine locales et les congrès, le programme ReMed a fait connaître sa vaste palette de prestations au sein des groupes cibles visés. Au plan international, la conférence EAPH a été l'occasion de présenter les prestations du réseau ReMed ainsi que les résultats de l'évaluation externe. La présentation a suscité un vif intérêt.

\section{2 prises de contact sur l'année écoulée}

Le nombre de prises de contact a augmenté de $10 \%$ au cours de l'année écoulée, passant à 162. Sur le seul mois de février, le réseau a réceptionné 20 demandes de soutien. Cette augmentation constante prouve combien il était urgent qu'un service tel que ReMed voie le jour. Sur ces 162 prises de contact, réceptionnées par les premiers répondants, 110 provenaient de Suisse alémanique, 50 de Suisse romande et 2 du Tessin. Les appelants étaient pour $60 \%$ des femmes et pour $40 \%$ des hommes, et la majorité d'entre eux (53\%) travaillent dans le secteur hospitalier. La problématique des «peurs» (craintes pour l'avenir, tournants dans la car- 
Raisons qui motivent la prise de contact (plusieurs réponses possibles)

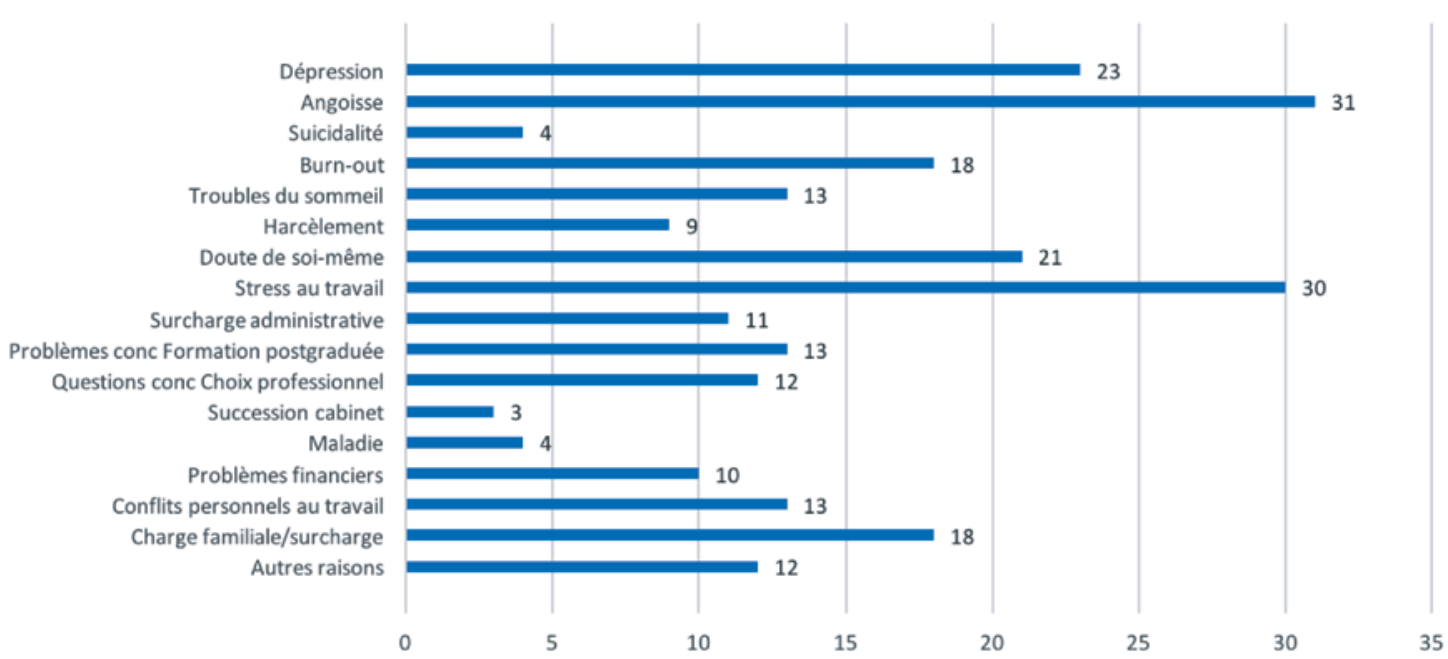

Figure 1: raisons de prendre contact avec ReMed (possibilité d'indiquer plusieurs causes).

rière, fermeture et transmission de cabinets, crainte de commettre une erreur médicale, etc.) était suivie par les problèmes de type "surcharge de travail» et "dépression" parmi les raisons les plus fréquentes pour contacter le réseau (cf. fig. 1).

\section{La santé du médecin comme prérequis d'une médecine de qualité}

Depuis 2020, la Haute école spécialisée bernoise en santé propose un CAS «Qualité en médecine pour le travail pratique auprès du patient». ReMed interviendra dans le cadre du module consacré à la santé des médecins et permettra aux étudiants d'approfondir leurs connaissances concernant la promotion de la santé et la prévention.

\section{Programme de mentoring «Coach my Career»}

À la fin de leurs études, et plus tard aussi, les jeunes médecins se trouvent face à des choix déterminants pour l'entier de leur carrière: Vais-je me diriger vers la recherche ou l'industrie? Ouvrir un cabinet ou débuter dans un hôpital ou une faculté? Il n'est pas rare qu'un jeune médecin ne trouve personne pour l'aiguiller dans ces décisions difficiles. C'est la raison pour laquelle la FMH, l'AMDHS, l'ASMAC, la MFE et la SWIMSA se sont mobilisées et ont mis sur pied - avec l'appui de l'ISFM - le programme de mentoring intergénérationnel «Coach my Career». Ce dernier se veut un complément aux offres proposées par les sociétés de mé- decine, les facultés et les hôpitaux, mais aussi par le réseau ReMed. Un échange aussi intéressant que constructif a d'ores et déjà eu lieu entre ReMed et "Coach my Career», pour faire en sorte que les deux programmes soient complémentaires et se soutiennent mutuellement.

\section{ReMed a le regret d'annoncer la perte d'un de ses membres fondateurs}

Nous avons eu la tristesse au cours de l'année écoulée de devoir prendre congé du Dr Peter Birchler, membre fondateur et membre actif de notre comité de direction. Avec Peter Birchler, nous perdons l'un des créateurs de notre réseau, auquel il avait su insuffler sa vive intelligence, sa clairvoyance et son infatigable engagement. Il restera dans nos mémoires.

\section{Comptes rendus d'expérience et documentation}

Venez nous rendre visite sur le site www.swiss-remed.ch, où vous trouverez d'intéressants comptes rendus d'expérience, de la documentation ainsi que diverses informations importantes sur le réseau de soutien.

Le programme national ReMed, son comité de direction et son réseau d'intervenants seront également là en 2020 pour les médecins en situation de crise. La permanence se tient à leur disposition 24 heures sur 24 et peut être jointe tant par téléphone, au 0800073633 , que par e-mail à l'adresse remed[at]hin.ch. 